\title{
VORBEMERKUNGEN DES HERAUSGEBERS
}

Die Vorlesung Hegels über die Philosophie der Weltgeschichte wechselt mit dieser Auflage das vierte Mal den Herausgeber, nachdem sie im Rahmen der "Vollständigen Ausgabe» der Werke Hegels "durch einen Verein von Freunden des Verewigten" zunächst 1837 von Eduard Gans, dann noch einmal 1840 von Karl Hegel und schließlich im Rahmen der "Kritischen Ausgabe" der "Philosophischen Bibliothek" von Georg Lasson, und zwar in drei Auflagen $(1917,1920,1930)$ dargeboten worden war. Von jedem der Herausgeber wurde der Text verändert, und der Letztgenannte, Georg Lasson, hat zwar seine Textgestalt bei der zweiten und dritten Auflage in der "Philosophischen Bibliothek" unverändert gelassen, aber er hat sie doch auf Grund nachträglicher Funde von Hand- und Nachschriften um einzelne wesentliche Stücke so erweitert, da $B$ sein Verfahren der Darbietung des Ganzen nicht mehr zu rechtfertigen war und deshalb zu einer abermaligen Durchsicht und Redaktion zwang.

Hegel hat diese Vorlesung vom Wintersemester 1822/23 an in zweijährigem Turnus, jeweils wöchentlich vierstündig, gehalten, also fünfmal, das letzte Mal im Wintersemester 1830/31; während dieser Jahre hatte sich ihm aber so vielfaches und vielseitiges neues empirisch-historisches Material ergeben, daß er im Wintersemester 1830/31 das Ganze nicht mehr zu bewältigen wußte und deshalb nur noch die "Philosophie der Weltgeschichte. Erster T e i l" ankündigte. Aber gerade dem großen historischen Materialreichtum waren die ersten Herausgeber in keiner Weise gerecht geworden; sowohl in der ersten als auch in der zweiten Auflage nimmt diese Hegelsche Vorlesung im Verhältnis zu den übrigen, die nicht in Buch- oder Paragraphenform wenigstens angelegt waren, nur einen schmalen Band ein. Es ist eins der wesentlichen Verdienste meines Vorgängers, diesem Übelstand abgeholfen und die Hegelsche "Philosophie der Weltgeschichte» erstmalig auf einen Stand ge- 
bracht zu haben, der ihrem gediegenen Gehalt, ihrer formellen Durchgliederung und insbesondere ihrer empirischen Stoffbreite einigermaßen entspricht.

Wie Lasson zu dieser Leistung auf Grund des erneuten Rückgangs zu den Quellen, den Hegelschen Handschriften und den Vorlesungs-Nachschriften und Ausarbeitungen seiner Hörer gelangte, darüber gibt er selbst in seinem Nachwort "Zur Herstellung des Textes" genügende Auskunft. Dasjenige, was aus diesem Nachwort auch für den Charakter der gegenwärtigen Auflage wichtig und wissenswert ist und also sowohl diese als die Lassonsche Textgestalt gegenüber derjenigen von Eduard Gans und Karl Hegel unterscheidet, ist am Schluß dieses Bandes wieder abgedruckt, das übrige dagegen, was Lasson bei dieser Gelegenheit vorbringt, gestrichen oder gekürzt worden. Seit Lassons Erstausgabe, die vielfach noch, dem damaligen Stadium der Hegel-Abneigung entsprechend, pädagogisch-didaktische Absichten in den Vordergrund stellt, sind die Ansprüche an die Darbietung von Hegel-Texten beträchtlich gewachsen. Wir erwarten heute, zumal hinsichtlich der Vorlesungs-, Nach- und Reinschriften, sowohl im Einzelnen eine strengere philologische Methode - denn manches, was von den Hegel-Schülern nachgeschrieben oder gar nach Notizen ausgewertet worden ist, stellt sich als ungenau oder abwegig heraus - als auch im Besonderen eine genaue Angabe der Herkunft der einzelnen Wendungen, Sätze und Absätze, die durchaus nicht immer "mosaikartig" zusammenpassen, sondern zum Teil in den verschiedenen Jahrgängen der Vorlesung erheblich voneinander abweichen, und schließlich ganz allgemein eine nähere Einsicht in den jeweiligen Aufbau der Gedanken der für die Gesamtredaktion des Textes benutzten Vorlesungen. Uber die philologisch-methodischen Konsequenzen, die sich aus diesem Charakter des Hegelschen Denkens für die Herausgabe seiner Vorlesungen ergeben, habe ich in meinem Vorwort zum Einleitungsband der "Geschichte der Philosophie» (Ph. B. XVa, 1940, 1944²) ausführlich berichtet. Diese Konsequenzen hätten sich eigentlich noch auf den vorliegenden Band umgestaltend auswirken müssen. Da es mir aber bisher noch nicht möglich war, die vorhan- 
denen und neu hinzugekommenen Quellen zu einer sachgerechteren Darbietung des Gesamttextes zu verwerten, mußte die bisherige Fassung, die Lasson aus den ihm vorliegenden Vorlesungsnachschriften gewonnen hatte, im wesentlichen beibehalten werden. Hinsichtlich dieser Partien war es mir nicht möglich, die Herkunft im einzelnen nach Quelle und Jahrgang festzu-. stellen. Es erübrigte sich damit auch, die Art ihrer Eingliederung in den Hegelschen Originaltext zu überprüfen bzw. zu ändern. Selbst die Zusätze, die Lasson nach Herstellung seines Textes aus zwei Vorlesungsnachschriften des Wintersemesters 1826/27 ermittelt und anhangsweise mitgeteilt hat, konnten nicht "eingearbeitet" werden, obwohl er für seinen Haupttext u. a. auch eine Nachschrift aus diesem Jahrgang benutzt hatte. Sie mußten abermals an den Schluß des Bandes gestellt werden.

Dennoch war es möglich, dem Band eine e in h e i t li c h e re Gestalt zu geben, und zwar e in mal von der Hegelschen Gliederung aus. Es erwies sich nämlich bei näherem Zusehen, daß die Einteilungsziffern am Rande des Hegelschen Hauptmanuskripts (des "Zweiten Entwurfs" in der vorliegenden Ausgabe), die Lasson nur als Gedächtnisstützen Hegels beim Vortrag gewertet hatte, doch dem methodischen Gesamtgang der Abhandlung weitgehend entsprachen, während die Lassonsche Durchgliederung vielfach von dem Material her, das er aus den Vorlesungs $\mathrm{n} \mathrm{a} \mathrm{ch} \mathrm{s} \mathrm{chriften} \mathrm{gewonnen} \mathrm{hatte,} \mathrm{bestimmt} \mathrm{ist.}$ Ich habe daher die Hegelsche Einteilung - z. T. unter Hinzufügung entsprechender Titel in eckigen Klammern - wiederhergestellt und da, wo Lasson, um des Andrangs des Stoffes Herr zu werden, weitere Untertitel für notwendig hielt, Abstände gelassen und Sternchen hinzugefügt. Ferner wurde im Zusammenhang hiermit der Lassonsche Lesartenapparat "Zur Textgestalt der Hegelschen Handschrift" (d. i. des "Zweiten Entwurfs«) aufgelöst; alles, was darin an irgend erwähnenswerten Hegelschen Untergliederungen, Bemerkungen und textlichen Besonderheiten aufgezählt ist, findet sich jetzt anmerkungsweise an den betreffenden Stellen im Hegeltext selbst. Die straffere Fassung des Bandes ergab sich $\mathrm{zum}$ andern dadurch, daß das Kapitel über "Die Arten der Geschichts- 
schreibung wieder in den Haupttext eingefügt werden konnte, aus dem Lasson es bei seiner Bearbeitung verwiesen hatte.

Bei der Neugestaltung des Textes der Einleitung im Jahre 1917 fand Lasson nur in der alten Ausgabe und in einigen Kollegnachschriften Anhaltspunkte dafür, daß Hegel seine Vorlesungen überhaupt jemals mit diesem Kapitel begonnen haben könnte. Die Sache selbst erschien ihm nicht genügend gesichert, zumal die einzige Handschrift, die ihm damals von Hegel selbst vorlag, eben der "Zweite Entwurf" vom Wintersemester 1830/31, ganz eindeutig für einen anderen Anfang sprach; und so setzte er dieses Kapitel kurzerhand an den Schluß des Bandes als erstes Stück einer "Besonderen Einleitung". Bald danach fanden sich zwar zu diesem Kapitel zwei einzelne Bogen von Hegels eigener Hand - der eine aus Züricher Privatbesitz, der andere aus dem Schiller-Museum zu Marbach - wieder ein, und aus den Daten, die Hegel selbst auf dem ersten vermerkt hatte, ergab sich, daß die Vorlesung zum mindesten zweimal, 1822 und 1828 , mit ihm eröffnet worden ist. Es war nun zwar weiterhin durchaus berechtigt, den zweiten, ausfürlicheren und auch endgültigeren Entwurf Hegels zur Einleitung als Grundlage der Textgestaltung beizubehalten und die Niederschriften "U'ber die Arten der Geschichtsschreibung " - ebenso wie die Ausführungen über den "Naturzusammenhang oder die geographische Grundlage der Weltgeschichte« - am Schluß zu belassen. Aber immerhin wäre es nun doch geraten gewesen, diese Niederschriften für eine textkritische Neufassung dieses Kapitels fruchtbar zu machen. Statt dessen ließ Lasson sie in der Form, die sich ihm aus seinen früheren Quellen und aus der alten Ausgabe ergeben hatte, im Rahmen seiner „Besonderen Einleitung" stehen und druckte gesondert davon die handschriftlichen Ausführungen Hegels als "Nachträge» ab. Es erschien also doppelt, an zwei verschiedenen Stellen. Erschwerend kam dabei hinzu, daß die Widerstände, die der zweite (Marbacher) Bogen der Entzifferungskunst des Herausgebers bereitete, nur mangelhaft überwunden wurden.

Der eigentliche Grund für die Behandlung des textkritischen Problems dieses Kapitels war freilich der, daß Lasson nicht 
bemerkt hatte, da $\beta$ die beiden neu aufgefundenen Bogen nicht etwa nur, wie er glaubte, mziemlich nahe, wohl höchstens durch einen halben Bogen getrennt«, zusammenhängen, sondern sich vielmehr w ö $\mathbf{r} \mathbf{l} \mathbf{i} \mathbf{c h}$, von einem Worte ("sein") zum nächsten ("Bewußtsein") aneinanderschließen. Ich habe die Nahtstelle im Text selbst bezeichnet. Durch diese genaue Zusammengehörigkeit, die übrigens auch Lassons Vorgänger nicht bemerkt hatten, ergibt sich mit den vermißten Schlußabsätzen zusammen, die nach wie vor aus den Kollegnachschriften bzw. den bisherigen Ausgaben ergänzt werden müssen, eine in sich geschlossene und durch Hegels eigenhändige Niederschrift fast durchgängig verbürgte Abhandlung, die den Namen eines "Ersten Entwurfs" schon deshalb zu Recht trägt, weil sie - nach dem Ausweis der Daten auf dem Züricher Bogen - wirklich diejenigen Gedanken bringt, mit denen Hegel seine erste Vorlesung über die Philosophie der Weltgeschichte eröffnet hat, und aus dem gleichen Grunde auch wieder die Stelle verdient, die die früheren Herausgeber ihr eingeräumt hatten. Im übrigen schließt, wie schon die jetzige "Inhaltsübersicht" zeigt, der "Zweite Entwurf" der Sache nach unmittelbar an den ersten an; ich habe es nur weder für nötig noch auch für richtig gehalten, dem dokumentarischen Charakter der Hegelschen Originalquellen irgend Abbruch zu tun, wie etwa Karl Hegel S. 11 f. seiner Ausgabe, um den direkten gedanklichen Zusammenhang beider Entwürfe zur Geltung zu bringen.

Der dritte neugefundene handschriftliche Bogen, den Lasson mitabdruckt, mußte hier wegfallen; sein Inhalt gehört in den Zusammenhang der "Orientalischen Welt" und damit in den zweiten Halbband der Ausgabe dieser Vorlesung.

Der Hegelsche Originaltext ist kursiv wiedergegeben worden, während die Zusätze aus den Nachschriften, die Lasson eingefügt hat, in Normaldruck gesetzt sind. Für die Mithilfe beim Lesen der Korrekturen und die Erneuerung der Register bin ich Herrn Dr. Rolf Bachem zu großem Dank verpflichtet.

Bonn, 15. Januar 1955.

Johannes Hoffmeister 